\title{
Measurement uncertainty of dissolution test of acetaminophen immediate release tablets using Monte Carlo simulations
}

\author{
Daniel Cancelli Romero, Felipe Rebello Lourenço*
}

\author{
Department of Pharmacy, Faculty of Pharmaceutical Sciences, Univerity of São Paulo, São Paulo, Brazil
}

\begin{abstract}
Analytical results are widely used to assess batch-by-batch conformity, pharmaceutical equivalence, as well as in the development of drug products. Despite this, few papers describing the measurement uncertainty estimation associated with these results were found in the literature. Here, we described a simple procedure used for estimating measurement uncertainty associated with the dissolution test of acetaminophen tablets. A fractionate factorial design was used to define a mathematical model that explains the amount of acetaminophen dissolved (\%) as a function of time of dissolution (from 20 to 40 minutes), volume of dissolution media (from 800 to $1000 \mathrm{~mL}$ ), $\mathrm{pH}$ of dissolution media (from 2.0 to 6.8), and rotation speed (from 40 to $60 \mathrm{rpm}$ ). Using Monte Carlo simulations, we estimated measurement uncertainty for dissolution test of acetaminophen tablets $(95.2 \pm 1.0 \%)$, with a $95 \%$ confidence level. Rotation speed was the most important source of uncertainty, contributing about $96.2 \%$ of overall uncertainty. Finally, it is important to note that the uncertainty calculated in this paper reflects the expected uncertainty to the dissolution test, and does not consider variations in the content of acetaminophen.
\end{abstract}

Keywords: Acetaminophen tablets/dissolution test/measurement uncertainty. Monte Carlo simulation.

\section{INTRODUCTION}

Analytical results (ARs) are the fundamental basis for the development of industry and science in modern society. In scientific research, ARs are used to compare experimental and control groups, test hypotheses, express relationships between variables, among other uses, in such a way that constitute the foundation for the discussion and conclusion of a particular study. In pharmaceutical industry, ARs are used to estimate income, assign a certain quality to materials, products or systems and compare them against certain internal or legal specifications or, among other applications. Usually, method validation, internal quality control protocols, arrangements of proficiency tests, and accreditation based on ISO/IEC 17025 are adopted as a kind of quality assurance system that allows assessment of accuracy and assertiveness of ARs (Ellison, Williams, 2012).

Despite this, an AR will never be able to be an absolute and perfect representation of the parameter

\footnotetext{
*Correspondence: F. R. Lourenço. Departamento de Farmácia, Faculdade de Ciências Farmacêuticas, Universidade de São Paulo. Av. Prof. Lineu Prestes, 580 - Bloco 13, CEP 05508-000 - São Paulo, SP, Brasil. Tel.: 55-11-3091-3649. E-mail: feliperl@usp.br
}

being measured. There will always be some uncertainty associated with the assertiveness of AR, even if all sources of uncertainty have been evaluated and corrected. This is because even the corrections applied have, by themselves, an associated uncertainty, not removing absolute uncertainty. Still, such a method of correction of uncertainty does not distinguish the variations from random effects, inherent to any experimental determination (Williams, 1998). Measurement uncertainty (MU) reflects a range of values that can be assigned to an AR using a specific methodology. It is not possible to correct the experimental results found for a true result and absolute through the MU (Ellison, Williams, 2012).

A measurement of uncertainty proposed in the Guide to the Expression of Uncertainty in Measurement (GUM) distinguishes both uncertainties from systematic and random effects (Ellison, Barwick, 1998a), by assessing the magnitude of the influence of some parameters on the measurement, and the determination of the impact of their uncertainties on AR. The first edition of the EURACHEM Guide entitled "Quantifying Uncertainty in Analytical Measurement" (Eurachem, 1995) was published in 1995. Focusing on various applications of analytical chemistry, from basic research up to routine analysis in major industries, 
the guide was well received by the analytical community (Williams, 1998). Five years later, EURACHEM/CITAC published the second version of the guide, emphasizing new concepts such as the integration of measuring uncertainty and in-house quality assurance procedures.

Although these approaches are different from those usually used in analytical chemistry (Barwick, Ellison, 1998, Ellison, Barwick, 1998b), the methodologies proposed in the GUM and EURACHEM/CITAC guides were applied in different areas such as, toxicological analysis (Eller et al., 2014), physical-chemical analysis (Leito et $a l ., 2002)$, and quantitative analysis of pharmaceuticals by microbiological (Ghisleni et al., 2014a; Lourenço et al., 2007; Lourenço, 2013), spectrophotometric (Saviano, Lourenço, 2013) and chromatographic methods (Ghisleni et al., 2014b; Saviano et al., 2015; Okamoto, Traple, Lourenço, 2013). Traple et al. (2014) published a review of MU applied in pharmaceutical analysis (Traple et al., 2014) describing the main sources of uncertainty such as sampling; instrumental effects; calibration mass and volumetric apparatus; purity of reagents and certified chemical substances; matrix effects and sample stability; analyst skills and training effects; environmental effects; random effects inherent to experimental determinations (Ellison, Williams, 2012).

In the third edition of the EURACHEM/CITAC guide, published in 2012 (Ellison, Williams, 2012), proficiency tests and application of Monte Carlo simulations were added as methods for the estimation of measurement uncertainty. The Monte Carlo simulation can be defined as randomly generating numbers by means of algorithms using probability distribution functions to obtain specific results on the process or object under study (Barat et al., 2006a; Barat et al., 2006), and it has been used in solving problems in several areas of science, including the chemical and pharmaceutical industry (Hammersley, Handscomb, 1964; Meimaroglou, Kiparissides, 2014). The main idea of the Monte Carlo simulation is that the extensive repetition of a random sampling process allows for obtaining a sufficiently large and random sample space for a statistical inference (Kroese et al., 2014). This random sampling is essential for simulating real mathematically modeled systems, and it also applies this statistical methodology to the concept of variability and measurement uncertainty, because it allows that the results generated in the output varies within a range determined by the algorithm as if they were random error fluctuations. The algorithms used in the Monte Carlo methods are simple, and have the ability to reduce the complexity of mathematically modeled systems (Kroese et al., 2014). This makes them applicable to, for example, processes of dissolution of controlled release pharmaceuticals (Barat et al., 2006b).

In the context of measurement uncertainty, the Monte Carlo simulation appeared first in 2008, as a document that describes the technique of propagation of distributions (JCGM, 2008). It is important to note that there are cases where the value of the measurement uncertainty will be relatively the same regardless of the methodology used. There are reports in the literature on the use of Monte Carlo simulation to ensure that the estimated probability functions for the factors of variability were indeed assertive, as a proof of concept of the classical methodology (Francisco, Saviano, Lourenço, 2014; Ghisleni et al., 2014b; Saviano, Madruga, Lourenço, 2015; Diaconu et al., 2015). The differences between the methodologies are evident in scenarios with a lack of normality, lack of linearity or even when a mathematical expression relating the results and factors of variability is too complex to be calculated, taking the simplifications, which lead to deviations in measurement of uncertainty. In these cases, the Monte Carlo simulation seems to be the most appropriate methodology to be used (Ellison, Williams, 2012).

The dissolution test is a procedure officially established in the pharmacopoeias of various countries, which expresses the amount of dissolved substance as a function of time, using a dissolution media with specified $\mathrm{pH}$, temperature and volume (ANVISA, 2010a; United States Pharmacopeia, 2012). Pharmacopeia monographs specify the device (basket, paddles or other) and appropriate conditions of dissolution test for each compound. The interests of the pharmaceutical community in the dissolution test began in the 1950s after observing that the disintegration profile of pharmaceutical dosage form did not explain the bioavailability profiles. Edwards was the first to report that if gastrointestinal absorption of a drug is fast, then the dissolution of pharmaceutical dosage form controls the bioavailability of the drug (Dokoumetzidis, Macheras, 2006), a preview of what would be proposed later by Amidon as the biopharmaceutical classification system (BCS) (Amidon et al., 1995). From the 1960 s to the 1980 s, the effects of dissolution on the bioavailability of drugs and the impact of formulations on bioavailability were widely studied (Dokoumetzidis, Macheras, 2006). Classic examples such as the ineffectiveness of tolbutamide and carbamazepine (Campagna et al., 1963; Meyer et al., 1992), and changes in the bioavailability of phenytoin and digoxin (Dokoumetzidis, Macheras, 2006). After investigation, these cases showed a correlation between changes in dissolution profiles and clinical toxicity or ineffectiveness. 
We found a few papers in the literature that described measurement uncertainty estimation associated with the dissolution test; however none of them assessed the contributions of dissolution test conditions of immediate release tablet dosage forms in the overall uncertainty. Here, we described a simple procedure used for estimating measurement uncertainty associated with the dissolution test of acetaminophen tablets based on Monte Carlo simulations.

\section{MATERIAL AND METHODS}

\section{Immediate-release tablets and reference standard}

Acetaminophen immediate-release tablets of $1000 \mathrm{mg}$ were purchased from Brazilian suppliers. An acetaminophen reference standard was provided by the United States Pharmacopeia.

\section{Instrumentation}

Dissolution tests were performed using a VanKel system (VanKel VK 7010) comprising a bath with six vessels and meeting the physical and mechanical specifications required by the USP chapter $<711>$ (United States Pharmacopeia, 2012). The instrument was mechanically calibrated using paddles. An analytical balance (Shimadzu, AUY220), a pHmeter (Gehaka, PG1800), a UV-visible spectrophotometer (Thermo Fisher Scientific, Evolution 201, Waltham, MA, USA) were also used. Dissolution media, sample and reference standard solutions were prepared using calibrated volumetric flasks and pipettes.

\section{Dissolution test}

Dissolution tests were performed as described in Brazilian Pharmacopeia (ANVISA, 2010b) with some modifications to study how dissolution conditions affect the amount of acetaminophen dissolved. A $2^{4-1}{ }_{\text {IV }}$ fractionate factorial design with a central point was adopted to evaluate how time of dissolution (A, from 20 to 40 minutes), volume of dissolution media ( $\mathrm{B}$, from 800 to $1000 \mathrm{~mL}$ ), $\mathrm{pH}$ of dissolution media (C, from 2.0 to 6.8 ), and rotation speed (D, from 40 to $60 \mathrm{rpm}$ ), affects the amount of acetaminophen dissolved. All dissolution tests were performed in 3 vessels (replicas) using paddle apparatuses. The amounts of acetaminophen dissolved were determined by employing UV absorption at the wavelength of maximum absorbance at about $243 \mathrm{~nm}$ on filtered portions of the diluted sample solution compared with a reference standard solution, using dissolution medium as blank, as described in Brazilian pharmacopeia (ANVISA, 2010b). The mean amounts of acetaminophen dissolved and their relative standard deviations (RSD) were calculated.

\section{Monte Carlo simulations}

A mathematical regression model was adjusted to explain the amount of acetaminophen dissolved as a function of time of dissolution (A), volume of dissolution media (B), $\mathrm{pH}$ of dissolution media (C), and rotation speed (D). A combination of linear, quadratic, and interaction terms were selected to provide higher predictive power. A Box-Cox transformation was used to improve homoscedasticity and normal distribution of residual values.

Monte Carlo simulations were performed with 20,000 random values of time of dissolution $(5,000$ random values, normal distribution, mean $=30$ minutes and standard deviation $=1$ minute), volume of dissolution media $(5,000$ random values, normal distribution, mean $=$ $900 \mathrm{~mL}$ and standard deviation $=5 \mathrm{~mL}$ ), $\mathrm{pH}$ of dissolution media (5,000 random values, normal distribution, mean $=5.80$ and standard deviation $=0.05)$, and rotation speed $(5,000$ random values, normal distribution, mean $=50 \mathrm{rpm}$ and standard deviation $=2 \mathrm{rpm}$ ).

\section{RESULTS AND DISCUSSION}

According to BCS, highly soluble and highly permeable drugs formulated into rapidly dissolving products do not need to be subjected to a profile comparison. Usually, a one-point test is required to confirm a release of $85 \%$ or more of the active pharmaceutical ingredient (API) within $15 \mathrm{~min}$. The impact of formulation composition in the dissolution is well established. However, slight changes in dissolution test conditions may impact the amount of API dissolved. Thus, this work focused on studying the effect of dissolution test conditions (such as time of dissolution, volume of dissolution media, $\mathrm{pH}$ of dissolution media, and rotation speed) in the amount of API dissolved and their contribution to overall uncertainty.

A fractionate factorial design was performed to statistically assess the magnitude of the influence of time of dissolution (A), volume $\mathrm{f}$ dissolution media (B), $\mathrm{pH}$ of dissolution media (C), and rotation speed (D) on the amount of acetaminophen dissolved. It was found that the data did not show a normal distribution; therefore a Box-Cox transformation was employed to statistically analyze the influence of inputs (A, B, C, and D) and 
their interactions $(\mathrm{AB}, \mathrm{AC}, \mathrm{AD}, \mathrm{BC}, \mathrm{BD}$, and $\mathrm{CD})$ on the output. The statistical results are shown in a Pareto chart (Figure 1). The inputs above the critical value (1.71) showed statistically significant influence on the dissolution. Thus, the rotation speed (D) was determined as the most significant factor, followed by the time of dissolution (A), and later by an interaction between $\mathrm{pH}$ and rotation speed (CD). The relative standard deviations (RSD) for all dissolution conditions were less than 3\%.

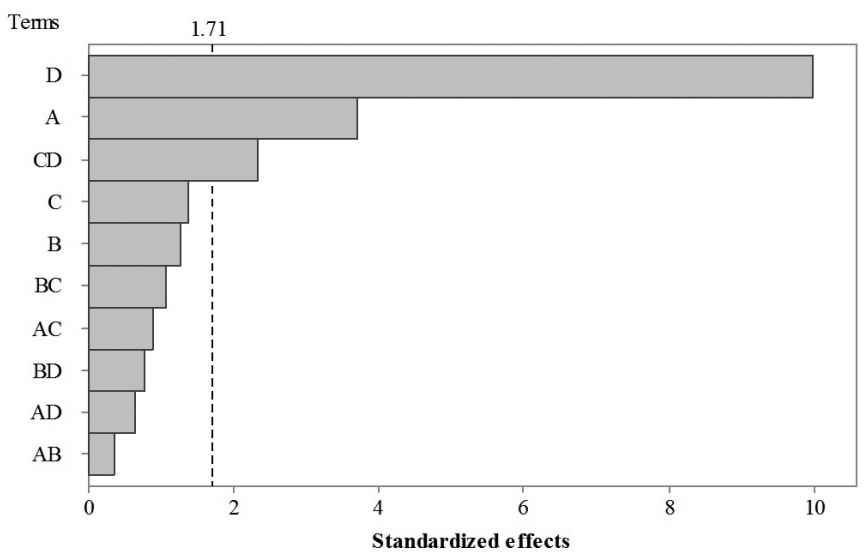

FIGURE 1 - Pareto chart for effects of time of dissolution (A), volume $\mathrm{f}$ dissolution media $(\mathrm{B}), \mathrm{pH}$ of dissolution media $(\mathrm{C})$, rotation speed $(\mathrm{D})$, and their interactions $(\mathrm{AB}, \mathrm{AC}, \mathrm{AD}, \mathrm{BC}, \mathrm{BD}$, and $\mathrm{CD}$ ) on the amount of acetaminophen dissolved.

The mathematical model obtained from the analysis of factorial experiment should be evaluated regarding its predictive power and its adjustment to the experimental results. The predictive coefficient of determination
( $\mathrm{R}^{2}$ pred) is calculated to assess the predictive power of the mathematical model. The $\mathrm{R}^{2}$ pred is calculated from the predicted residual error sum of squares (PRESS) statistic, and it provides a number that is directly proportional to the capacity of the mathematical model to predict the output with certainty. It is very important that the mathematical model has high predictive ability, since it will be used to calculate the Monte Carlo simulation outputs from the random inputs.

The adjustment of a mathematical model usually is evaluated based on two parameters: $R^{2}$ and $R^{2}$ adj. $R^{2}$ is proportional to total variability explained by the model, and it will be close to $100 \%$ when the model is most adjusted to the experimental data. However, $\mathrm{R}^{2}$ may not be the most reliable variable as it may increase due to an increased number of non-significant factors included in the model. Thus, $\mathrm{R}^{2}$ adj is often used to compare mathematical models, since it precisely adjusts to the number of factors included in the model.

The Pareto chart of the complete model includes the linear terms of time of dissolution (A), volume $f$ dissolution media (B), $\mathrm{pH}$ of dissolution media (C), rotation speed $(\mathrm{D})$, and their interactions $(\mathrm{AB}, \mathrm{AC}, \mathrm{AD}$, $\mathrm{BC}, \mathrm{BD}$, and $\mathrm{CD}$ ) are shown in Figure 1. Despite a high $\mathrm{R}^{2}$ value $(83.45 \%)$, the complete model was not well adjusted due to the inclusion of insignificant factors. A second model including the linear terms of time of dissolution (A), volume $\mathrm{f}$ dissolution media (B), $\mathrm{pH}$ of dissolution media (C), rotation speed (D), and the interaction between $\mathrm{pH}$ and rotation speed (CD) was adjusted. According to the Pareto chart (Figure 2) and ANOVA results (Table I), the most significant factors were rotation speed (D), time

TABLE I - Analysis of variance (ANOVA) results of the amount of acetaminophen dissolved (\%) as a function of time of dissolution (A), volume of dissolution media (B), $\mathrm{pH}$ of dissolution media (C), and rotation speed (D)

\begin{tabular}{lccccc}
\hline Source & d.f. & SS & MS & F & p-value \\
\hline Model & 5 & 0.026204 & 0.026204 & 26.55 & $0.000^{* *}$ \\
Linear & 4 & 0.024847 & 0.024847 & 31.47 & $0.000^{* *}$ \\
A & 1 & 0.002836 & 0.002836 & 14.37 & $0.001^{* *}$ \\
B & 1 & 0.000311 & 0.000311 & 1.58 & 0.219 \\
C & 1 & 0.000432 & 0.000432 & 2.19 & 0.150 \\
D & 1 & 0.021268 & 0.021268 & 107.75 & $0.000^{* *}$ \\
Interactions & 1 & 0.001180 & 0.001180 & 5.98 & $0.021^{*}$ \\
$\quad$ CD & 1 & 0.001180 & 0.001180 & 5.98 & $0.021^{*}$ \\
Error & 30 & 0.005921 & 0.000197 & & \\
Total & 35 & 0.032125 & & & \\
\hline
\end{tabular}

Legend: d.f.: degrees of freedom, SS: Sum of Squares, MS: Mean of Squares, F: calculated F statistics, and p-value: *significant (p-value $<0.05)$ and ${ }^{* *}$ very significant $(\mathrm{p}$-value $<0.01)$. 
TABLE II - Regression equation and coefficients of determination $\left(\mathrm{R}^{2}, \mathrm{R}^{2} \mathrm{adj}\right.$, and $\mathrm{R}^{2}$ pred $)$ of the amount of acetaminophen dissolved $(\%)$ as a function of time of dissolution (A), volume of dissolution media (B), $\mathrm{pH}$ of dissolution media (C), and rotation speed (D)

\begin{tabular}{cccc}
\hline Regression equation & $\mathbf{R}^{2}$ & $\mathbf{R}^{2}$ adj & $\mathbf{R}^{2}$ pred \\
\hline $\begin{array}{c}(\%-1) /\left(\lambda \times \mathrm{g}^{(\lambda-1)}\right)=-0.2132+0.001087 \times \mathrm{A}+0.000029 \times \mathrm{B} \\
-0.01284 \times \mathrm{C}+0.001146 \times \mathrm{D}+0.000292 \times \mathrm{C} \times \mathrm{D}\end{array}$ & $81.57 \%$ & \multirow{2}{*}{$78.50 \%$} & $73.22 \%$ \\
\hline
\end{tabular}

$\lambda=17$ (from Box-Cox transformation) and $\mathrm{g}=0.9420$ (geometric mean).

of dissolution (A), and the interaction between $\mathrm{pH}$ and rotation speed (CD), respectively. Regression equation and coefficients of determination $\left(\mathrm{R}^{2}, \mathrm{R}^{2} \mathrm{adj}\right.$, and $\left.\mathrm{R}^{2} \mathrm{pred}\right)$ of this model is shown in Table II. A Box-Cox transformation $(\lambda=17$, and geometric mean $g=0.941974)$ was used to normalize the amount of acetaminophen dissolved (output). The main effect and interaction effect plots are shown in Figure 3.

Response surface plot was obtained based on the mathematical model adjusted in order to show the effect of time of dissolution (A) and rotation speed (D) (Figure 4) in the amount of acetaminophen dissolved (\%), using volume (B) and $\mathrm{pH}(\mathrm{C})$ of dissolution media fixed at $900 \mathrm{~mL}$ and 5.8, respectively. According to Figure 4, the amount of acetaminophen dissolved was more susceptible to time of dissolution (from 20 to 40 minutes) when a low rotation speed (40 rpm) is used. Moreover, at high rotation

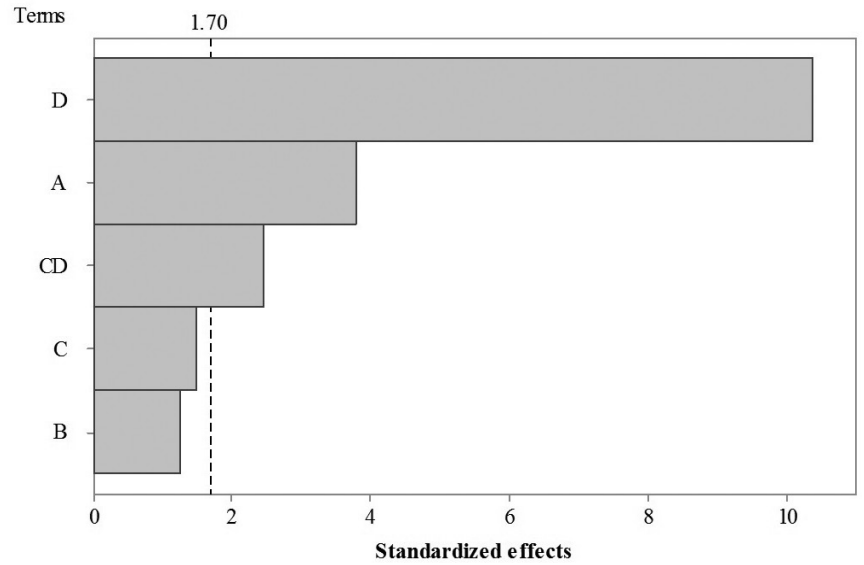

FIGURE 2 - Pareto chart for effects of time of dissolution (A), volume $\mathrm{f}$ dissolution media (B), $\mathrm{pH}$ of dissolution media (C), rotation speed (D), and the interaction of $\mathrm{pH}$ and rotation speed (CD) on the amount of acetaminophen dissolved.

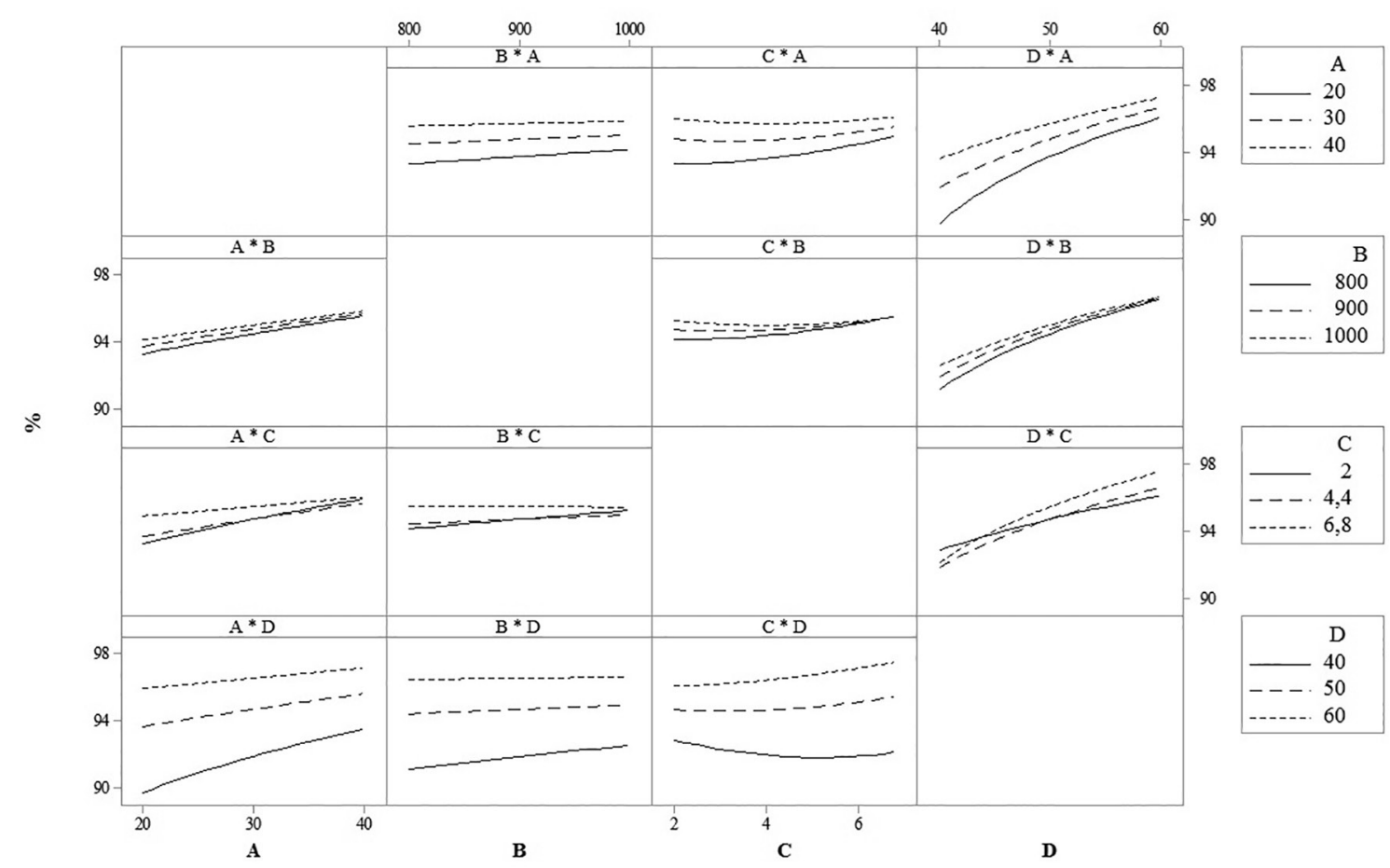

FIGURE 3 - Main effects and interactions plots for the effect of time of dissolution (A, from 20 to 40 minutes), volume f dissolution media (B, from 800 to $1000 \mathrm{~mL}$ ), $\mathrm{pH}$ of dissolution media (C, from 2.o to 6.8), rotation speed (D, from 40 to $60 \mathrm{rpm}$ ), and their interactions $(\mathrm{AB}, \mathrm{AC}, \mathrm{AD}, \mathrm{BC}, \mathrm{BD}$, and $\mathrm{CD})$ on the amount of acetaminophen dissolved. 
speed (60 rpm), the amount of acetaminophen dissolved was almost not affected by time of dissolution (from 20 to 40 minutes). Similarly, Figure 5 shows the response surface plot of the amount of acetaminophen dissolved $(\%)$ as a function of time of dissolution (A) and $\mathrm{pH}$ of dissolution media (C), with volume of dissolution media (B) and rotation speed (D) fixed at $900 \mathrm{~mL}$ and $50 \mathrm{rpm}$, respectively. At low rotation speed $(40 \mathrm{rpm})$, the amount of dissolved acetaminophen slightly decreased as the $\mathrm{pH}$ increased. On the other hand, the amount of dissolved acetaminophen slightly increased as the $\mathrm{pH}$ increase, at high rotation speed (60 rpm.).

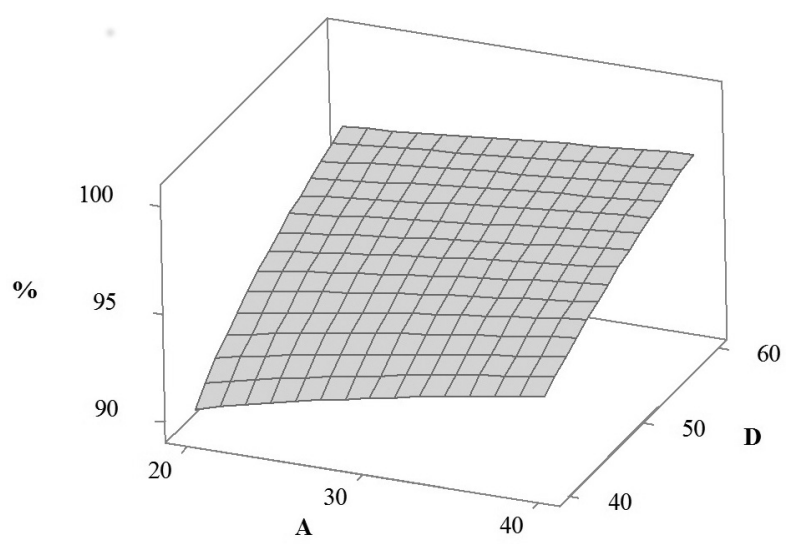

FIGURE 4 - Response surface plot of the effect of time of dissolution (A) and rotation speed (D) on the amount of acetaminophen dissolved (\%).

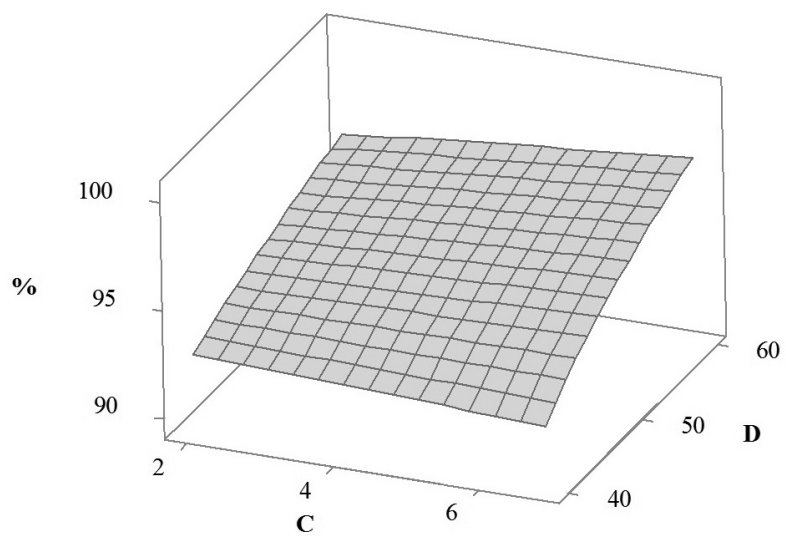

FIGURE 5 - Response surface plot of the effect of $\mathrm{pH}$ of dissolution media (C) and rotation speed (D) on the amount of acetaminophen dissolved (\%).

It did not escape our notice that $\mathrm{pH}$, the input factor with the widest range $(\mathrm{C}$, from 2.0 to 6.8$)$ did not affect the amount of acetaminophen dissolved. $\mathrm{pH}$ alters the ionization of organic molecules, which alters the solubility of active pharmaceutical ingredient (API) in dissolution media. The non-significant effect of $\mathrm{pH}$ in the amount of acetaminophen dissolved may be explained due to its $\mathrm{pKa}$ (9.46), as well as the $\log \mathrm{S}$ and $\log \mathrm{P}$ curves as functions of $\mathrm{pH}$. In other words, solubility of acetaminophen will be affected only in alkaline $\mathrm{pH}$. Thus, solubility modifications in the $\mathrm{pH}$ range adopted in our study were not expected.

On the other hand, rotation speed significantly affects the amount of acetaminophen dissolved. This may be explained due to the effects of the hydrodynamic environment on the tablet dissolution rate (Kamba et al., 2003; Wu, Kildsig, Gahly, 2004). Rotation speed was even more significant than time of dissolution. We believe this may be explained because acetaminophen is a high solubility drug (BCS Class I) (Kalantzi et al., 2006). Thus, the amount of acetaminophen dissolved in the range of time adopted in our study was close to the plateau level. Considering the importance of the hydrodynamic properties on the dissolution, the absence of volume as a factor of influence on the amount of acetaminophen dissolved could be questioned. However, the volume of dissolution media $(900 \mathrm{~mL})$ was large enough for the variation proposed in our study (from 800 to $1000 \mathrm{~mL}$ ) to be not significant, since acetaminophen is a high solubility drug (Granber, Rasmuson, 1999). Because of the high solubility of acetaminophen, even under drastic changes in the significant factors in the dissolution test, minimal dissolution reached around $90 \%$, which is considerably above the minimum of $80 \%$ in $30 \mathrm{~min}$ (ANVISA, 2010b; United States Pharmacopeia, 2012). Therefore, the dissolution test for acetaminophen tablets resists variations in experimental conditions.

The Monte Carlo simulation applied in estimating measurement uncertainty requires that the output response has a mathematical relationship with the inputs or sources of uncertainty. In addition, the inputs must have a probability density function, which can be 'obtained from existing information about the process or evaluated experimentally. Random values of the density of probability of each input will be sampled and configured as mathematical expressions that will generate a simulated output result. This process is repeated a high number of times, generating a distribution of simulated results that will be used to determine uncertainty (Meyer, 2007; Ellison, Williams, 2012).

Monte Carlo simulations were performed with 5,000 random values of time of dissolution $(5,000$ random values, normal distribution, mean $=30$ minutes and standard deviation $=1$ minute), volume of dissolution media $(5,000$ random values, normal distribution, mean $=$ $900 \mathrm{~mL}$ and standard deviation $=5 \mathrm{~mL}$ ), $\mathrm{pH}$ of dissolution media (5,000 random values, normal distribution, mean $=5.80$ and standard deviation $=0.05)$, and rotation speed $(5,000$ random values, normal distribution, mean $=50 \mathrm{rpm}$ 
and standard deviation $=2 \mathrm{rpm}$ ). The $\mathrm{pH}$ of dissolution media used in the central point of fractionate factorial design was 4.4. Despite this, we performed Monte Carlo simulations using a dissolution media $\mathrm{pH}$ of 5.8 , which is the $\mathrm{pH}$ used in the monograph of Brazilian pharmacopeia (ANVISA, 2010b).

The estimation of measurement uncertainty associated with the dissolution test would be much more difficult using the Eurachem/Citac Guidelines or spreadsheet methods. This is because the effects of dissolution conditions (such as time of dissolution, volume of dissolution media, $\mathrm{pH}$ of dissolution media, and rotation speed) on the amount of acetaminophen dissolved are not mathematically established. Measurement uncertainty estimation would be easier using other approaches such as method validation and repeatability/reproducibility studies. However, these approaches do not provide information of individual contributions of each source of uncertainty (Diaconu et al., 2015; Francisco, Saviano, Lourenço, 2016; Takano et al., 2017). Thus, we estimated the measurement uncertainty by Monte Carlo simulations performed using the regression equation of the amount of acetaminophen dissolved (\%) as a function of time of dissolution (A), volume of dissolution media (B), $\mathrm{pH}$ of dissolution media (C), and rotation speed (D) (Table II).

This approach is in accordance with the principle of determination of measurement uncertainty described in the EURACHEM Guide (Ellison, Williams, 2012). From the simulated results of dissolution (output), we obtained a histogram plot (Figure 6) with normal distribution, characterized by its mean (95.2\%) and standard deviation $(0.5 \%)$. Expanded uncertainty may be obtained by the multiplication of standard deviation $(0.5 \%)$ by a coverage factor $(\mathrm{k}=2)$, which correspond to a $95 \%$ confidence interval (from 94.2 to $96.2 \%$ ).

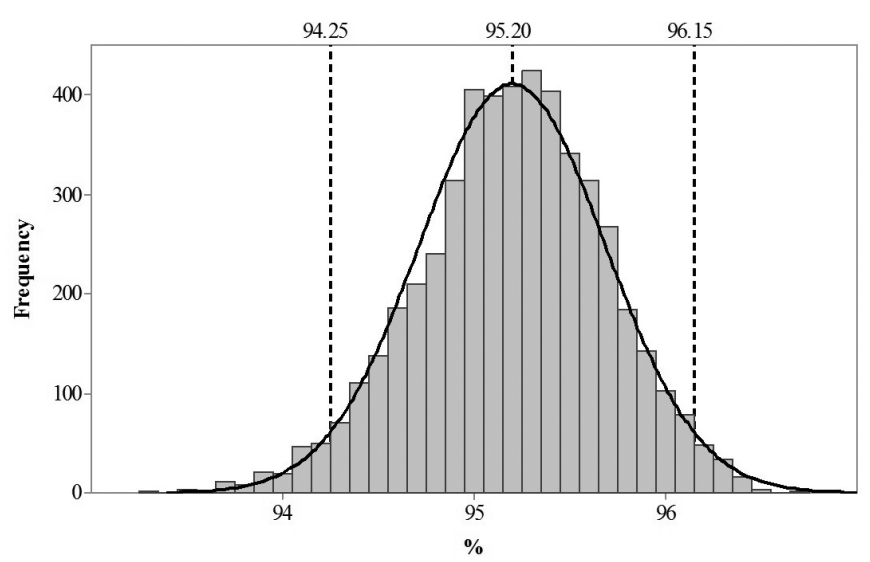

FIGURE 6 - Histogram plot obtained from 5,000 Monte Carlo simulations of the amount of acetaminophen dissolved.
The ANOVA (Table I) and Pareto's Chart (Figure 2) indicate that time of dissolution (A), rotation speed (D), and the interaction between $\mathrm{pH}$ of dissolution media and rotation speed (CD) were significant terms of regression equation (Table II). However, considering the individual contribution of each source of uncertainty, the rotation speed was the most important source of uncertainty, contributing about $96.2 \%$ of overall uncertainty (Figure 7). The other factors contribute with virtually insignificant uncertainties. It is important to note that the uncertainty calculated in this paper reflects the expected uncertainty to the dissolution test, and does not consider variations in the content of acetaminophen. Pendrill raises some critical points on how to differentiate the uncertainty of measurement inherent in the manufacturing process. This question must be observed in the implementation of measurement uncertainty using EURACHEM and GUM methods (Pendrill, 2014).

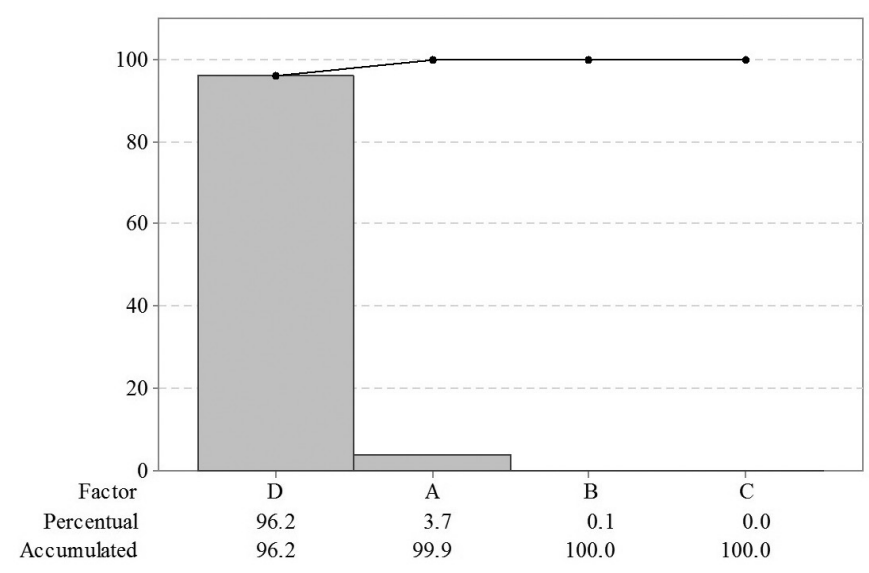

FIGURE 7 - Pareto chart of the contribution of time of dissolution (A), volume of dissolution media (B), $\mathrm{pH}$ of dissolution media (C), and rotation speed (D) to overall uncertainty.

\section{CONCLUSION}

The main factors of variability for the dissolution of acetaminophen were rotation speed, time of dissolution, and the interaction between $\mathrm{pH}$ and rotation speed. Using Monte Carlo simulations, we estimated measurement uncertainty for a dissolution test of acetaminophen tablets $(95.2 \pm 1.0 \%)$, with a $95 \%$ confidence level. Amounts of acetaminophen dissolved using the experimental range proposed in our study were found to be considerably above the minimum of $80 \%$ in $30 \mathrm{~min}$. Finally, it is important to note that the uncertainty calculated in this paper reflects the expected uncertainty to the dissolution test and does not consider variations in the content of acetaminophen. 


\section{REFERENCES}

Agência Nacional de Vigilância Sanitária. ANVISA. Teste de Dissolução. In: Farmacopeia Brasileira. Cap.5.1.5. Brasília: ANVISA; 2010a. v.1, p. 66-73.

Agência Nacional de Vigilância Sanitária. ANVISA. Paracetamol Comprimidos. In: Farmacopeia Brasileira. Brasília: ANVISA; 2010b. v.2, p. 1190-1192.

Amidon GL, Lennernäs H, Shah VP, Crison JR. A theoretical basis for a biopharmaceutical drug classification: the correlation of in vitro drug product dissolution and in vivo bioavailability. Pharm Res. 1995;12(3):413-420.

Barat A, Ruskin HJ, Crane M. Probabilistic models for drug dissolution. Part 1. Review of Monte Carlo and stochastic cellular automata approaches. Simul Model Pract Theory. 2006a;14(7):843-856.

Barat A, Ruskin HJ, Crane M. Probabilistic models for drug dissolution. Part 2. Modelling a soluble binary drug delivery system dissolving in vitro. Simul Model Pract Theory. 2006b;14(7):857-873.

Barwick VJ, Ellison SLR. Estimating measurement uncertainty using a cause and effect and reconciliation approach Part 2. Measurement uncertainty estimates compared with collaborative trial expectation. Anal Comm. 1998:35(11):377-383.

Campagna FA, Cureton G, Mirigiam, RA, Nelson E. Inactive prednisone tablets. J Pharmac Scien. 2006;52:605-606.

Diaconu I, Mirea CM, Ruse E, Balea AM. Uncertainty estimation fir acetaminophen spectrophotometric determination after the transport through bulk liquid membrane. UPB Sci Bull Series B. 2015:77(1):41-52.

Dokoumetzidis A, Macheras P. A century of dissolution research: from noyes and whitney to the biopharmaceutics classification system. Inter J Pharmac. 2006;321(1-2): 1-11.

Eller SCWS, Flaiban LG, Paranhos BAPB, Da Costa JL, Lourenço FR, Yonamine M. Analysis of 11-nor-9-carboxy$\Lambda^{9}$-tetrahydrocannabinol in urine samples by hollow fiberliquid phase microextraction and gas chromatography-mass spectrometry in consideration of measurement uncertainty. Forensic Toxicol. 2014;32(2):282-291.
Ellison SLR, Barwick VJ. Estimating measurement uncertainty: reconciliation using a cause and effect approach. Accred Qual Assur. 1998a;3(3):101-105.

Ellison SLR, Barwick VJ. Using validation data for ISO measurement uncertainty estimation Part 1. Principles of an approach using cause and effect analysis. Analyst. 1998b;123(6):1387-1392.

Ellison SLR, Williams A. Eurachem/CITAC guide: quantifying uncertainty in analytical measurement. 3rd. ed. [London]: Eurachem; 2012. [citado 2016 Aug 13]. Disponível em: <www. eurachem.org>.

Eurachem Guide. Quantifying uncertainty in analytical measurement. Laboratory of the Government Chemist. London: BSI; 1995.

Francisco FL, Saviano AM, Lourenço FR. In vitro therapeutic equivalence of ciprofloxacin ophtalmic drugs based on measurement uncertainty and Monte Carlo simulations. Lat Am J Pharm. 2014;32(2):237-244.

Francisco FL, Saviano AM, Lourenço FR. Measuring uncertainty using different approaches: a case study for acetaminophen quantification. J AOAC Int. 2016;99(3):612-617.

Granberg RA, Rasmuson AC. Solubility of paracetamol in pure solvents. J Chem Eng Data. 1999;44(6):1391-1395.

Ghisleni DDM, Okamoto RT, Amaral CMO, Lourenço FR, Pinto TJA. New microbiological assay for determination of caspofungin in the presence of its degradation products and its measurement uncertainty. J AOAC Int. 2014a;97(3):908-912.

Ghisleni DDM, Okamoto RT, Lourenço FR, Pinto TJA. Validation and measurement uncertainty of an UPLC-PDA stability indication method applied to degradation kinetics of caspofungin. Curr Pharmac Anal. 2014b;10(3):193-202.

Hammersley M, Handscomb DC. The general nature of Monte Carlo methods. In: Monte Carlo Methods. London: Methuen \& Co; 1964.p. 1-9.

Joint Committee for Guides in Metrology. JCGM. Evaluation of measurement data - Supplement 1 to the "Guide to the Expression of Uncertainty in Measurement" - Propagation of distributions using a Monte Carlo method. 2008. [citado 2016 Ago 14]. Disponível em: $<$ http://www.bipm.org/en/publications/ guides/\#gum>. 
Kalantzi L, Reppas C, Dressman JB, Amidon GL, Junginger HE, Midha KK, Shah VP, Stavchansky SA, Barends DM. Biowaiver monographs for immediate release solid oral dosage forms: Acetaminophen (paracetamol). J Pharm Sci. 2006;95(1):1-4.

Kamba M, Seta Y, Takeda N, Hamaura T, Kusai A, Nakane H, Nishimura K. Measurement of agitation force in dissolution test and mechanical destructive force in disintegration test. Int J Pharm. 2003;250(1):99-109.

Kroese DP, Brereton T, Taimre T, Botev ZI. Why the Monte Carlo method is so important today. WIREs Comput Stat. 2014;6(6):386-392.

Leito I, Strauss L, Koort E, Pihl V. Estimation of uncertainty in routine $\mathrm{pH}$ measurement. Accred Qual Assur. 2002;7(6):242249.

Lourenço FR. Uncertainty measurement of microbiological assay for apramycin soluble powder. Lat Am J Pharm. 2013;32(5):640-645.

Lourenço FR, Kaneko TM, Pinto TJA. Evaluating measurement uncertainty in the microbiological assay of vancomycin from methodology validation data. J AOAC Int. 2007;90(5):13831386.

Meimaroglou D, Kiparissides C. Review of Monte Carlo methods for the prediction of distributed molecular and morphological polymer properties. Ind Eng Chem Res. 2014;53(22):8963-8979.

Meyer MC, Straughn AB, Jarvi EJ, Wood GC, Pelsor FR, Shah VP. The bioinequivalence of carbamazepine tablets with a history of clinical failures. Pharm Res. 1992;9(12):1612-1616.

Meyer VR. Measurement uncertainty. J Chromat A. 2007;1158(1-2):15-24.
Okamoto RT, Traple MAL, Lourenço FR. Uncertainty in the quantification of metronidazole in injectable solution and its application in the assessment of pharmaceutical equivalence. Curr Pharm Anal. 2013;9(4):355-362.

Pendrill LR. Using of measurement uncertainty in decision-making and conformity assessment. Metrologia. 2014;51:S206-S218.

Saviano AM, Lourenço FR. Uncertainty evaluation for determining linezolid in injectable solution by UV spectrophotometry. Measurement. 2013;46(10):3924-3928.

Saviano AM, Madruga ROG, Lourenço FR. Measurement uncertainty of a UPLC stability indicating method for determination of linezolid in dosage forms. Measurement. 2015;59:1-8

Takano DEN, Reis PRS, Singh A, Lourenço FR. Estimation of uncertainty for measuring desloratadine in tablets formulation using UV spectrophotometry. Measurement. 2017 (in press).

Traple MAL, Saviano AM, Francisco FL, Lourenço FR. Measurement uncertainty in pharmaceutical analysis and its application. J Pharm Anal. 2014;4(1):1-5.

United States Pharmacopeia. <771> Dissolution. Rockville: United States Pharmacopeia, 2011.

Williams A. Introduction to measurement uncertainty in chemical analysis. Accred. Qual. Assur. 1998;3(3):92-94.

Wu Y, Kildsig DO, Gahly ES. Effect of hydrodynamic environment on tablet dissolution rate. Pharm Dev Tech. 2004;9(1):25-37.

Received for publication on $21^{\text {st }}$ November 2016 Accepted for publication on $09^{\text {th }}$ February 2017 